\title{
Corrigendum: Effects of Anthocyanin Supplementation on Serum Lipids, Glucose, Markers of Inflammation and Cognition in Adults With Increased Risk of Dementia - A Pilot Study
}

Anne Katrine Bergland ${ }^{1,2 *}$, Hogne Soennesyn ${ }^{1}$, Ingvild Dalen ${ }^{3}$, Ana Rodriguez-Mateos ${ }^{4}$, Rolf Kristian Berge ${ }^{5}$, Lasse Melvaer Giil ${ }^{6}$, Lawrence Rajendran ${ }^{7}$, Richard Siow ${ }^{8}$, Michele Tassotti ${ }^{4,9}$, Alf Inge Larsen ${ }^{2,10}$ and Dag Aarsland ${ }^{1,7}$

OPEN ACCESS

Edited by:

Joseph Baur,

University of Pennsylvania,

United States

Reviewed by:

Indika Edirisinghe,

Illinois Institute of Technology,

United States

Wieslaw Wiczkowski,

Institute of Animal Reproduction and

Food Research (PAN), Poland

*Correspondence:

Anne Katrine Bergland

anne.katrine.bergland@sus.no

Specialty section:

This article was submitted to

Genetics of Aging,

a section of the journal

Frontiers in Genetics

Received: 09 March 2021

Accepted: 21 April 2021

Published: 31 May 2021

Citation:

Bergland AK, Soennesyn H, Dalen I,

Rodriguez-Mateos A, Berge RK,

Giil LM, Rajendran L, Siow R,

Tassotti M, Larsen Al and Aarsland D

(2021) Corrigendum: Effects of

Anthocyanin Supplementation on

Serum Lipids, Glucose, Markers of Inflammation and Cognition in Adults With Increased Risk of Dementia - A Pilot Study. Front. Genet. 12:678504.

doi: 10.3389/fgene.2021.678504
${ }^{1}$ Centre for Age-Related Medicine, Stavanger University Hospital, Stavanger, Norway, ${ }^{2}$ Department of Clinical Science, University of Bergen, Bergen, Norway, ${ }^{3}$ Section of Biostatistics, Department of Research, Stavanger University Hospital, Stavanger, Norway, ${ }^{4}$ Department of Nutritional Sciences, Faculty of Life Sciences and Medicine, School of Life Course Sciences, King's College London, London, United Kingdom, ${ }^{5}$ The Lipid Research Group, Department of Clinical Science, University of Bergen, Bergen, Norway, ${ }^{6}$ Department of Internal Medicine, Haraldsplass Deaconess Hospital, Bergen, Norway, ${ }^{7}$ UK Dementia Research Institute, Institute of Psychiatry, Psychology \& Neuroscience, King's College London, London, United Kingdom, ${ }^{8}$ School of Cardiovascular Medicine and Sciences, British Heart Foundation Centre of Research Excellence, Faculty of Life Sciences and Medicine, King's College London, London, United Kingdom, ${ }^{9}$ Department of Food \& Drug, University of Parma, Parma, Italy, ${ }^{10}$ Department of Cardiology, Stavanger University Hospital, Stavanger, Norway

Keywords: mild cognitive impairment, $\mathrm{MCl}$, anthocyanins, lipids, inflammation markers

\section{A Corrigendum on}

Effects of Anthocyanin Supplementation on Serum Lipids, Glucose, Markers of Inflammation and Cognition in Adults With Increased Risk of Dementia - A Pilot Study

by Bergland, A. K., Soennesyn, H., Dalen, I., Rodriguez-Mateos, A., Berge, R. K., Giil, L. M., et al. (2019). Front. Genet. 10:536. doi: 10.3389/fgene.2019.00536

In the original article, there was a mistake in Table 2 as published.

Due to a typographical error, the authors supplied incorrect $p$-values in Table 2, which resulted in subsequent errors in the results and discussion sections and the abstract. The main change is that there is no statistically significant between-group difference for $\triangle$ RANTES (difference from baseline to study end). Also, concerning $\triangle \mathrm{MCP}-1$ and $\Delta$ fasting glucose statistically significant between-group differences emerged.

All of the incorrect data in Table 2 have been revised and are written in bold italics.

The corrected Table 2 appears below.

Consequently, there was an error in the abstract:

"CCL-5/RANTES [regulated on activation, normal T-cell expressed and secreted (RANTES)]" should be "monocyte chemoattractant protein (MCP-1) and fasting glucose."

A correction has been made to the abstract under subsection "Results":

Results: There was a significant difference between groups for monocyte chemoattractant protein (MCP-1) and fasting glucose. In addition, total cholesterol and triglycerides were significantly increased in the AG. Improvements in memory and executive test scores were observed. No adverse effects were reported. 
TABLE 2 | Changes from baseline to 16 weeks follow-up in serum variables, for participants with supplementation (active) and for control participants.

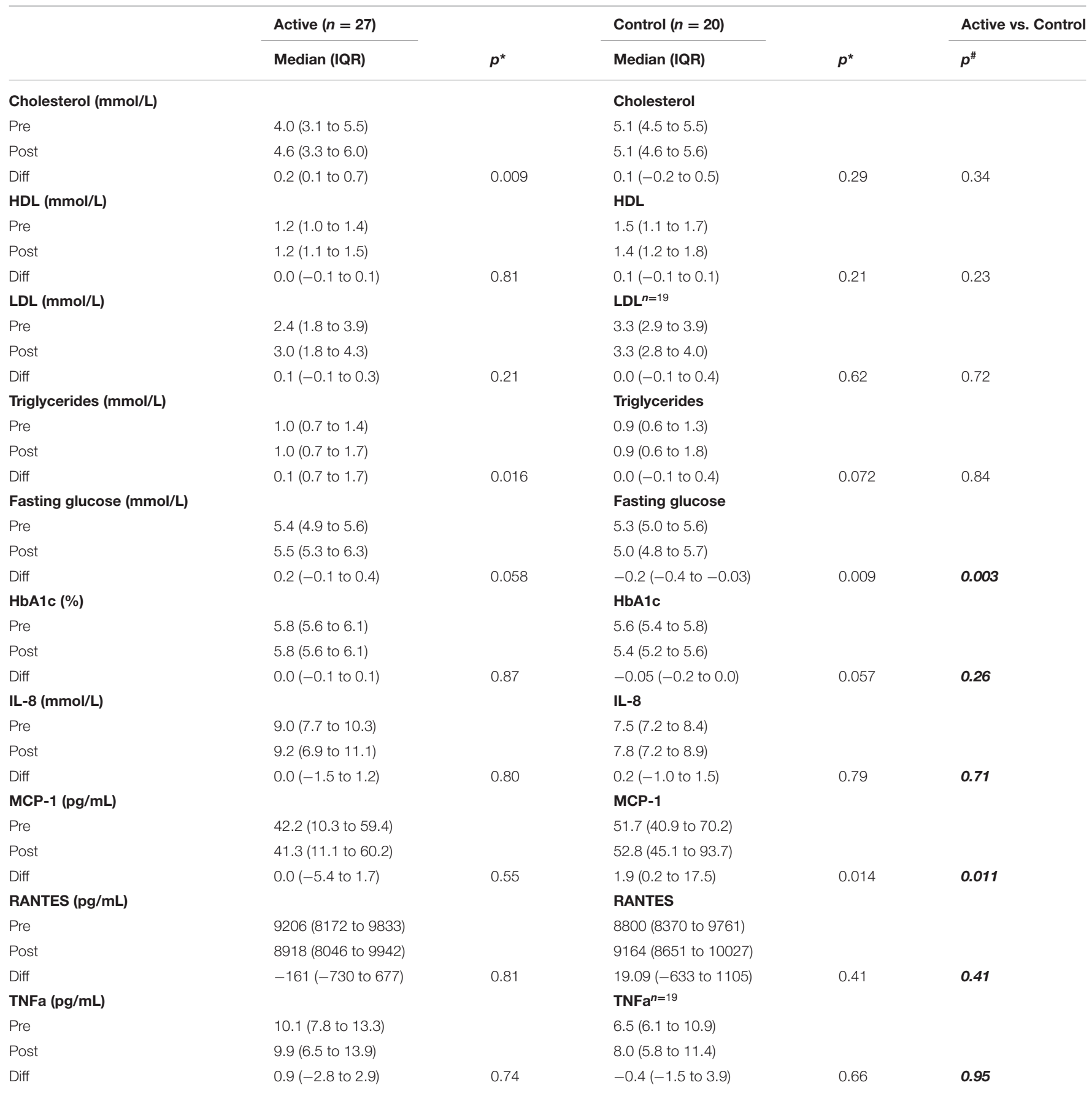

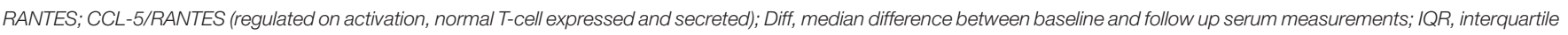

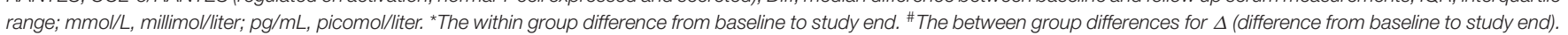

Also, there was an error in the result section:

"The only significant between-group difference was for $\triangle$ RANTES (difference from baseline to study end) which decreased in the supplementation group and increased in the NC group" should be "The only significant between-group difference was for difference were for $\triangle \mathrm{MCP}-1$ (difference from baseline to study end) $(p=0.011)$ and $\Delta$ fasting glucose $(p=0.003)$."
A correction has been made to the Section: Results, Paragraph 3:

The only significant between-group difference was for difference were for $\triangle \mathrm{MCP}-1$ (difference from baseline to study end) $(p=0.011)$ and $\Delta$ fasting glucose $(p=0.003)$. (Table 2$)$.

Finally, there were errors in the discussion section:

"There was a non-significant decrease in serum levels of RANTES in the AG and a non-significant increase in 
the NC during the study period. However, the betweengroup difference in $\Delta$ serum levels of RANTES was statistically significant." should be "There was a nonsignificant increase in serum levels of MCP-1 in the AG and a significant increase in the NC during the study period. The between-group difference in $\Delta$ serum levels of MCP-1 was statistically significant."

"In addition; There was a significant between-group difference for $\triangle$ RANTES, although anthocyanin supplementation did not significantly reduce RANTES in the AG. Still, our results are consistent with similar findings in a randomized, double-blind trial in hypercholesterolemic individuals consuming purified anthocyanins for 24 weeks (Song et al., 2014), and in a parallel-designed, placebo-controlled trial (Karlsen et al., 2007)." should be "As there was a significant between-group difference for $\triangle \mathrm{MCP}-1$, our results are partly consistent with findings in a randomized, double-blind trial in hypercholesterolemic individuals consuming purified anthocyanins for 24 weeks (Song et al., 2014), and in a parallel-designed, placebo-controlled trial (Karlsen et al., 2007).”

Corrections have been made to section: Discussion, Paragraphs 2 and 6:

Our findings are somewhat inconclusive. While some cognitive improvements were observed in the AG, there were no significant changes in serum levels of some risk factors for dementia; i.e., fasting glucose, HbAlc or pro-inflammatory cytokines. There was a non-significant increase in serum levels of MCP-1 in the AG and a significant increase in the NC during the study period. The between-group difference in 1 serumlevels of MCP-1 was statistically significant.

Regarding the inflammation markers, RANTES promotes activation and migration of leukocytes and mediates neuroinflammation and brain microvascular dysfunction (Appay and Rowland-Jones, 2001; Dénes et al., 2010; Yilmaz and Granger, 2010). As there was a significant between-group difference for 1MCP-1, our results are partly consistent with findings in a randomized, double-blind trial in hypercholesterolemic individuals consuming purified anthocyanins for 24 weeks (Song et al., 2014), and in a parallel-designed, placebo-controlled trial (Karlsen et al., 2007). Other studies did not report a reduction of pro-inflammatory mediators after anthocyanin supplementation (Hassellund et al., 2013; Kent et al., 2015). Therefore, the anti-inflammatory effect of anthocyanins and the potential to reduce neuroinflammation and brain microvascular dysfunction associated with cognitive decline in adults at risk of dementia (Grammas, 2011) should be studied in larger randomized studies.

The authors apologize for these errors and state that they do not change the scientific conclusions of the article in any way. The original article has been updated.

Copyright (c) 2021 Bergland, Soennesyn, Dalen, Rodriguez-Mateos, Berge, Giil, Rajendran, Siow, Tassotti, Larsen and Aarsland. This is an open-access article distributed under the terms of the Creative Commons Attribution License (CC BY). The use, distribution or reproduction in other forums is permitted, provided the original author(s) and the copyright owner(s) are credited and that the original publication in this journal is cited, in accordance with accepted academic practice. No use, distribution or reproduction is permitted which does not comply with these terms. 